\title{
Identification and functional characterization of SIDronc in Spodoptera littoralis
}

\author{
Hao Liu ${ }^{\text {Corresp., }}{ }^{1}$, Ke Zhou ${ }^{1}$, Zhouning Yang ${ }^{1}$ \\ ${ }^{1}$ State Key Laboratory of Virology, Modern Virology Research Center, College of Life Sciences, Wuhan University, Wuhan, China \\ Corresponding Author: Hao Liu \\ Email address: liuhao901001@whu.edu.cn
}

Background. Apoptosis is responsible for eliminating damaged and virus-infected cells, regulating normal cell turnover, and maintaining the immune system's development and function. Caspases play a vital role in both mammal and invertebrate apoptosis.

Spodoptera littoralis is a generalist insect herbivore that is one of the most destructive pests in tropical and subtropical areas and attacks a wide range of commercially important crops. Although S. littoralis is a model organism in the study of baculovirus infection, its apoptotic pathway has not been explored. Methods. We cloned a new caspase gene named sldronc in S. littoralis using Rapid Amplification of cDNA Ends (RACE). We then measured caspase activity on synthetic caspase substrates and S. littoralis' effector caspase. SIDronc's function in the apoptotic pathway and its interaction with caspase inhibitors were also tested in SL2 cells. Results. We found that the initiator caspase SIDronc cleaved and activated effector caspase in S. littoralis. SIDronc overexpression induced apoptosis in SL2 cells, and SIdronc knockdown decreased apoptosis induced by UV irradiation in SL2 cells. Our results indicate that SIDronc acts as an apoptotic initiator caspase in S. littoralis. Additionally, we found that processed forms of SIDronc increased in the presence of N-terminally truncated S. littoralis inhibitors of apoptosis (SIIAP) and that SIDronc was inhibited by P49. This study contributes to the further understanding of $S$. littoralis' apoptotic pathway and may facilitate future studies on baculovirus infectioninduced apoptosis. 
1 Identification and functional characterization of

2 SIDronc in Spodoptera littoralis

3

4 Hao Liu*, Ke Zhou, Zhouning Yang

6 State Key Laboratory of Virology, Modern Virology Research Center, College of Life Sciences,

7 Wuhan University, Wuhan, China

9 Corresponding Author:

10 Hao Liu

11 Wuhan, Hubei, China

12 Email address: liuhao901001@whu.edu.cn

Abstract

Background. Apoptosis is responsible for eliminating damaged and virus-infected cells,

regulating normal cell turnover, and maintaining the immune system's development and subtropical areas and attacks a wide range of commercially important crops. Although $S$. been explored. 
22 Methods. We cloned a new caspase gene named sldronc in S. littoralis using Rapid

Amplification of cDNA Ends (RACE). We then measured caspase activity on synthetic caspase substrates and S. littoralis' effector caspase. S1Dronc's function in the apoptotic pathway and its interaction with caspase inhibitors were also tested in SL2 cells.

Results. We found that the initiator caspase SlDronc cleaved and activated effector caspase in $S$. littoralis. SIDronc overexpression induced apoptosis in SL2 cells, and Sldronc knockdown decreased apoptosis induced by UV irradiation in SL2 cells. Our results indicate that SIDronc acts as an apoptotic initiator caspase in S. littoralis. Additionally, we found that processed forms of SIDronc increased in the presence of N-terminally truncated S. littoralis inhibitors of apoptosis (SIIAP) and that SIDronc was inhibited by P49. This study contributes to the further understanding of $S$. littoralis' apoptotic pathway and may facilitate future studies on baculovirus infection-induced apoptosis.

\section{Introduction}

Apoptosis is a form of strictly controlled and programmed cell death that eliminates impaired and virus-infected cells (Clem 2001; Duprez et al. 2009). Apoptosis plays a vital role in normal cell turnover and the proper development and function of the immune system (Elmore 2007). Apoptosis mainly proceeds through the intrinsic and extrinsic pathways, both of which converge during caspase activation (Degterev et al. 2003; Hay \& Guo 2006; Tait \& Green 2010). Caspases are cysteine-dependent, aspartate-directed proteases that cleave numerous specific target sites in cellular proteins after upstream signal activation (Wallach et al. 2016). According to their localization in the apoptotic pathway and biological functions, caspases can be divided into 
43

44

several groups, including initiator caspases, effector caspases, inflammatory caspases, and caspases without a known function (Cohen 1997; Galluzzi et al. 2016; Li \& Yuan 2008).

Caspases are synthesized as inactive zymogens, or pro-caspases. Pro-caspases have a prodomain in the $\mathrm{N}$-terminal and a conserved catalytic domain in the C-terminal, which consists of a large subunit and a small subunit. During apoptosis, the pro-caspase is cleaved between the prodomain and large subunit, the cleavage between the large subunit and the small subunit is not essential for caspase activation. A heterodimer is made up of a large subunit and a small subunit, and an active unit of tetramer is formed by two heterodimers. Initiator caspase generally has a long prodomain containing caspase recruit domains (CARDs) or death effector domains (DEDs). CARDs and DEDs can combine with adapter proteins located in the upstream of initiator caspase in the apoptotic pathway via their homologous motifs. However, effector caspase has a short prodomain without a DED or CARD (Lo et al. 2015; Salvesen \& Abrams 2004). Initiator caspase is activated by dimerization that is facilitated by recruiting caspases to oligomeric activation platforms that assemble following an apoptotic signal (Donepudi et al. 2003; Dorstyn $\&$ Kumar 2008; Pop et al. 2006). The activated initiator caspase activates effector caspase through proteolytic cleavage (Pop \& Salvesen 2009).

There are seven caspases in Drosophila melanogaster, a model organism in the study of insect apoptosis. Dronc and Dredd are initiator caspases with prodomains that have one CARD or two DEDs, respectively; Drice, Dcp-1, Decay, and Damm are effector caspases (Cooper et al. 2009; Kumar \& Doumanis 2000). Dronc acts as an upstream caspase in the intrinsic pathway and its activation mechanism resembles that of mammalian caspase-9 (Dorstyn et al. 1999; Muro et al. 
64 2004). Lepidopteran caspases are classified into six clades. Lep-Caspase-1, Lep-Caspase-2, and

Lep-Caspase-3 are the supposed effector caspases, and Lep-Caspase-5 and Lep-Caspase- 6 are the supposed initiator caspases. Dronc belongs to the Lep-caspase-5 clade (Courtiade et al. 2011). Previous studies have reported on Dronc homologs from several Lepidopteran insects, including Bombyx mori (BmDronc), Lymantria dispar (LdDronc), and Spodoptera frugiperda (SfDronc) (Huang et al. 2013; Kitaguchi et al. 2013; Suganuma et al. 2011).

Apoptosis is regulated by multiple cellular proteins, including inhibitor of apoptosis (IAP) which function as the last line of defense against caspase-mediated apoptosis. IAP can inhibit caspases by directly binding to them through baculoviral IAP repeat (BIR) domains or ubiquitylating caspases with the RING domain following binding (Deveraux et al. 1997; Ditzel et al. 2008; Roy et al. 1997). IAP need to be processed by caspases in order to work. For example, DIAP1 requires caspase-mediated cleavage to function, drICE cleaves $20 \mathrm{~N}$-terminal amino acids to activate DIAP1's ability to suppress apoptosis, and DIAP1's C-terminal is degraded by the Nend rule degradation pathway (Ditzel et al. 2003; Yan et al. 2004). The N-end rule pathway is a proteolytic system that depends on proteasome, and recognizes and degrades proteins that have N-degrons (Gibbs et al. 2014; Tasaki et al. 2012).

Apoptosis is also regulated by inhibitors in baculovirus. P35 in Autographa californica multiple nucleopolyhedrovirus (AcMNPV) and P49 in Spodoptera littoralis nucleopolyhedrovirus (SpliNPV) are two baculoviral apoptosis inhibitors. Generally, baculoviral apoptosis inhibitor P49 inhibits the caspase activity of initiator caspases and baculoviral apoptosis inhibitor P35 inhibits the caspase activity of effector caspases (Jabbour et al. 2002; Zoog et al. 2002). 
85

86

S. littoralis is a generalist insect herbivore that targets a wide range of commercially important crops, including cotton, rice, maize, and potato (Lee \& Anstee 1995). S. littoralis is distributed across Africa, the Mediterranean region, and the Near East, and is one of the most destructive pests in tropical and subtropical areas (Hill 1987). SL2 cells derived from S. littoralis and Sf9 cells derived from $S$. frugiperda are often used when studying baculovirus infection and apoptosis (Mialhe et al. 1984). Compared to Sf9 cells, SL2 cells undergo apoptosis and produce very low levels of polyhedrin when infected with AcMNPV (Chejanovsky \& Gershburg 1995), suggesting that SL2 and Sf9 cells have different apoptosis mechanisms. However, several years have passed since the first study on effector caspase, S1-caspase-1, and S. littoralis inhibitors of apoptosis (SIIAP) was published (Liu et al. 2005; Vilaplana et al. 2007). Since then, no initiator caspases have been identified and very few articles have expounded on the apoptosis mechanism of S. littoralis. In this study, we identified SIDronc, an initiator caspase in S. littoralis. We analyzed the amino acid sequences of SIDronc, tested its biochemical character, and verified its function in SL2 cells. This study contributes to the further understanding of S. littoralis' apoptotic pathway and may facilitate future research on baculovirus infection-induced apoptosis.

\section{Materials \& Methods}

\section{Cells}

SL2 cells were kindly gifted by Professor Nor Chejanovsky, Agricultural Research Organization, Volcani Center, Israel. SL2 cells were cultured using Grace's insect medium (Invitrogen, Carlsbad, CA, USA) at $27{ }^{\circ} \mathrm{C}$ in a biochemical incubator, and $10 \%(\mathrm{v} / \mathrm{v})$ heat-inactivated fetal bovine serum (FBS, Gibco, Waltham, MA, USA) was added to the insect medium. 
106

107

108

109

110

111

112

113

114

115

116

117

118

119

120

121

122

123

124

125

126

\section{Antibodies}

Rat-derived monoclonal antibodies against His-tag, Flag-tag, HA-tag, and $\beta$-actin (Proteintech,

Rosemont, IL, USA) were diluted in block buffer (1:5000) to be used for western blot analysis.

We diluted rabbit-derived polyclonal antibody against Sf-caspase-1 (also provided by Professor

Nor Chejanovsky), which can recognize full-length, large subunits of Sf-caspase-1 and S1-

caspase-1, 1:1000 in block buffer to be used for western blotting. A polyclonal antibody against

SIDronc, which can recognize full-length and large subunits of S1Dronc, was produced using a

SIDronc fragment purified in E. coli as an antigen to immunize rabbits. We produced a

polyclonal antibody against SfIAP, which can also recognize full-length, cleaved SIIAP, using a

SfIAP fragment purified in E. coli as an antigen to immunize rabbits.

\section{Cloning S. littoralis dronc}

We first cloned Sldronc as a partial sequence using the designed primers according to the alignment of dronc homologs from S. frugiperda (Sfdronc) and Spodoptera litura (Sl-caspase-5).

The sequence contained an intact open reading frame (ORF) that was highly similar to Sfdronc's. To determine Sldronc's full length, we designed primers for Rapid Amplification of cDNA Ends (RACE) according to Sldronc's obtained partial sequence (Table 1). We used the SMARTerTM RACE cDNA Amplification Kit (Clontech, Mountain View, CA, USA) in the 5' and 3' RACE to amplify Sldronc's coding sequence and untranslated regions (UTR). The purified PCR products were ligated into the pCR-II vector (TA Cloning ${ }^{\circledR}$ Kit, Invitrogen, Carlsbad, CA, USA) and plasmids extracted from several positive colonies were sequenced. Our sequencing results showed an assembled sequence containing a 1,338-bp ORF with a 216-bp 5'UTR and a 397-bp 3'UTR. We 
127

128

129

130

131

132

133

134

135

136

137

138

139

140

141

142

143

144

145

146

147

named this ORF Sldronc and cloned it into the pCR-II vector from S. littoralis cDNA. Plasmids extracted from several positive colonies were sequenced, confirming the sequence information from RACE.

\section{Constructing plasmids}

We constructed plasmid pET28a-S1Dronc-C-His-expressing SIDronc with a His-tag at the Cterminal by ligating SIDronc's ORF into Nco I and Hind III sites in vector pET-28a. The plasmids pET28a-S1-caspase-1-C-His and pET28a-P49-(GS) ${ }_{3}-\mathrm{C}-H i s$ were used for S1-caspase-1 and P49 expression containing a C-terminal His-tag in E. coli. Plasmid pIE1-SIDronc-C-Flag was used for SIDronc overexpression containing a C-terminal Flag-tag in SL2 cells. Plasmid pIE1-N-HA-SIDronc was used for SIDronc overexpression containing an N-terminal HA-tag in SL2 cells. Plasmid pIE1-P49-C-His was used for P49 overexpression with a C-terminal His-tag in SL2 cells. Plasmid pIE1-SIIAP-C-Flag was used for SIIAP overexpression with a C-terminal Flag-tag in SL2 cells. We constructed S1Dronc C310A, S1-caspase-1 C178A, S1IAP D87A, and SIIAP N88G by introducing point mutation into wild type plasmids using site-directed mutagenesis PCR. The forward and reverse primers are listed in Table 2. DNA sequencing was used to verify all constructed plasmids.

\section{Purifying recombinant proteins}

We cultured E. coli BL21 (DE3)/pLysS cells transfected by plasmid expressing interest protein or vector in LB medium with $50 \mu \mathrm{g} / \mathrm{mL}$ kanamycin until it reached an $\mathrm{OD}_{600 \mathrm{~nm}}$ concentration between 0.4 and 0.6 . The interest protein expression was induced by $0.2 \mathrm{mM}$ isopropyl- $\beta$-Dthiogalactopyranoside (IPTG) and the cells were cultured for another $2 \mathrm{~h}$ at $200 \mathrm{rpm}$. We used 20 
148

\section{Caspase activity assay} ${ }^{\circ} \mathrm{C}$.

mM imidazole solution with $1 \%$ detergent Triton X-100 and protease inhibitor (Roche, Basel, Switzerland) to resuspend BL21 cells after centrifugation, then sonicated the suspension for 4 seconds with 6 second intervals for a total of $30 \mathrm{~min}$, and then 10-min intervals between every 10 min sonication. The BL21 cell lysate was centrifugated at $14,000 \mathrm{~g}$ for 30 min at $4{ }^{\circ} \mathrm{C}$, and $\mathrm{Ni}$ NTA high affinity resin (Genscript, Piscataway, NJ, USA) was incubated with the supernatant according to the manufacturer's instructions. We used $20 \mathrm{mM}, 50 \mathrm{mM}$, and $80 \mathrm{mM}$ imidazole solution to wash off the resin and $250 \mathrm{mM}$ imidazole solution to elute the interest proteins. After western blotting and Coomassie staining analyses, we stored the purified interest proteins at -80

\section{SDS-PAGE and western blotting}

SDS-PAGE samples were prepared by mixing purified proteins or cell lysates with $5 \times$ SDS loading buffer, and then heating the mixture in boiling water for $10 \mathrm{~min}$. We detected proteins using Coomassie blue staining or western blotting on a PVDF membrane (Millipore, Burlington, MA, USA). We used 5\% milk or BSA in TBST to block the membranes for $1 \mathrm{~h}$ at room temperature, diluted the primary antibodies in block buffer, and incubated the membranes for 1 h. After washing three times with TBST, we incubated the horseradish peroxidase (HRP)conjugated secondary antibodies (Thermo Fisher, Waltham, MA, USA) that had been diluted in block buffer with the membranes for $1 \mathrm{~h}$. LAS 4000 (Fujifilm, Tokyo, Japan) was used to detect the protein-primary antibody-secondary antibody-HRP complex after incubating the membranes with chemiluminescent substrate (Millipore). 
169 In order to analyze the substrate preference of recombinant SIDronc purified in E. coli, we used

17013 kinds of fluorogenic synthetic caspase substrates: Ac-VEID-AFC, Ac-LETD-AFC, Ac-IETD-

171 AFC, Ac-LEHD-AFC, Ac-AEVD-AFC, Ac-WEHD-AFC, Ac-DEVD-AFC, Ac-YVAD-AFC,

172 Ac-DMQD-AFC, Ac-LEED-AFC, Ac-IEPD-AFC, Ac-VDVAD-AFC, and Ac-LEVD-AFC

173 (MP). We also measured the effects of Sl-caspase-1 and the cell lysates on Ac-DEVD-AFC. We

174 mixed purified caspase, incubate, or cell lysate with the appropriate fluorogenic substrate in Na-

175 Citrate buffer (1 M Na-Citrate, $50 \mathrm{mM}$ Tris-base, $10 \mathrm{mM}$ DTT, 0.05\% CHAPS, pH 7.4), and

used $20 \mu \mathrm{M}$ substrate in $100 \mu \mathrm{L}$ of each test mixture (Pop et al. 2008). The relative fluorescence

unit was detected at $37^{\circ} \mathrm{C}$ every $2 \mathrm{~min}$ for $2 \mathrm{~h}$ after $30 \mathrm{~min}$ of incubation at $37^{\circ} \mathrm{C}$. We used the

data to calculate the maximum slope of each curve and generated the graph using GraphPad

Prism 6.

\section{Transfection}

SL2 cells were transfected using the method previously described (Summers \& Smith 1987).

SL2 cells were seeded into a culture plate at a cell abundance of about $70 \%$. After the attachment

period, we removed the medium and added a mixture of plasmid, transfection buffer ( $25 \mathrm{mM}$

HEPES, $\mathrm{pH} 7.1,140 \mathrm{mM} \mathrm{NaCl}$, and $125 \mathrm{mM} \mathrm{CaCl}_{2}$ ), and Grace's insect medium dropwise into

the well with the SL2 cells. The cells were maintained at $27{ }^{\circ} \mathrm{C}$ for 4 hours before we replaced

the transfection mixture with fresh Grace's medium. We used $3.0 \mu \mathrm{g}$ plasmid per well in the 12-

well plate.

\section{UV irradiation}

We treated the SL2 cells with UV irradiation by placing the cell culture plate on a 
190

191

192

193

194

195

196

197

198

199

200

201

202

203

204

205

206

207

208

209

210

transilluminator for $45 \mathrm{~min}$. Twenty-four hours after UV treatment, the cells were harvested and lysed for further analysis.

\section{Cell lysate preparation}

SL2 cells were harvested via centrifugation of the culture medium at $1,000 \mathrm{~g}$ for $10 \mathrm{~min}$. Cell lysates were prepared using the following procedure. Lysis buffer $(200 \mathrm{mM}$ Tris-HCl pH 7.4, $150 \mathrm{mM} \mathrm{NaCl}, 1 \mathrm{mM}$ EDTA, and 1\% NP-40) mixed with protease inhibitor (Roche) was used to suspend the cells (Yang et al. 2016). After three freeze-thaw cycles, we centrifuged the suspension at $14,000 \mathrm{~g}$ at $4{ }^{\circ} \mathrm{C}$ for $10 \mathrm{~min}$, and then removed the supernatants as cell lysates for analysis. When harvesting the apoptotic SL2 cells, we first harvested cells at 3,000 $\mathrm{g}$ at $4{ }^{\circ} \mathrm{C}$ for $10 \mathrm{~min}$, and then centrifuged the supernatant to collect the apoptotic bodies at $14,000 \mathrm{~g}$ at $4{ }^{\circ} \mathrm{C}$ for $20 \mathrm{~min}$. The harvested cells and apoptotic bodies were gathered for cell lysate preparation using the routine method.

\section{Gene knockdown}

We cloned the DNA fragments of interest using primers (Table 3 ) that contained $\mathrm{T} 7$ promoter in the 5 ' end and plasmids or cDNAs that contained desire sequences as templates. RNAs were transcribed in vitro using the cloned DNA fragments. We removed the template DNA by adding TURBO DNase, and we extracted and purified the RNAs using phenol-chloroform. To obtain dsRNAs, the RNA products were slowly cooled to room temperature after incubation at $95{ }^{\circ} \mathrm{C}$ for 2 min. We used the NanoDrop One (Thermo Fisher) to determine dsRNA qualities and concentrations. We placed $1 \mu \mathrm{g}$ dsRNA in each $6 \times 10^{5}$ SL2 cell to knock down the desire gene. The dsRNA transcribed from the $g f p$ gene was utilized as the negative control. 


\section{Results}

\section{SIDronc sequence analysis}

213 We acquired a novel caspase cDNA, Sldronc, using RT-PCR on total mRNA from SL2 cells as a 214 template. The predicted protein encoded by the Sldronc ORF contained 445 amino acids (about $21551 \mathrm{kDa}$ ), which included a $15 \mathrm{kDa}$ prodomain, a $24 \mathrm{kDa}$ large subunit, and a $12 \mathrm{kDa}$ small 216 subunit. The alignment of SIDronc's predicted amino acid sequence with Dronc homologs

217 SfDronc, BmDronc, AeDronc, and DmDronc showed that SIDronc had 87\%, 54\%, 27\%, and 218 24\% consistency ratios with SfDronc, BmDronc, AeDronc, and DmDronc, respectively (Fig. 1).

219 SIDronc also had a predicted typical caspase catalytic site sequence $\mathrm{Q}^{308} \mathrm{MCRG}^{312}$ (Fig. 1). The predicted secondary structure of SIDronc contained a series of $\alpha$-helices and $\beta$-sheets that were

highly conserved with Droncs in other insects (Watt et al. 1999)Fig. 1). SlDronc possesses a long prodomain containing a CARD, E128 is predicted to be a cleavage site between prodomain and large subunit, and D338 is predicted to be the cleavage site between the large and small subunit according to the verified cleavage sites in other Droncs (Fig. 1).

\section{SIDronc phylogenetic analysis}

Our phylogenetic analysis of SIDronc and 30 chosen caspases in several insects (Table 4)

suggested that the closest relatives of SIDronc belong to the Dronc homolog clade, they are S1-

caspase-5, Se-caspase-5, SfDronc, and Ha-caspase-5 in lepidopteran insects (Fig. 2).

SIDronc autocatalytic cleavage in $E$. coli

Caspases usually autoprocess when brought into close proximity with each other according to the “induced-proximity” model (Riedl \& Shi 2004; Salvesen \& Dixit 1999). Consistent with this 
232 model, we detected three major bands (about $52 \mathrm{kDa}, 38 \mathrm{kDa}$, and $13 \mathrm{kDa}$ ) by western blotting

233 when recombinant SIDronc with C-terminal His-tag was expressed and purified in E. coli (Fig.

234 3). The 52-kDa band was predicted to be the full length of S1Dronc plus the C-terminal His-tag

235 (Full length + His). We predicted that the $38-\mathrm{kDa}$ band was the fragment consisting of the

236 prodomain and large subunit (Pro+LS). The 13-kDa band matched the supposed fragment

237 consisting of the small subunit and the C-terminal His-tag ( $\mathrm{SS}+\mathrm{His})$. The two forms of cleaved

238 bands indicated that SIDronc underwent autocatalytic cleavage when expressed and purified in

239 E. coli. In order to confirm whether the autocleavage of wild type SIDronc relies on its caspase

240 activity, we mutated cysteine (C) at the 310 site to alanine (A) in S1Dronc's predicted catalytic

241 site. Western blotting detected that the catalytic site mutant S1Dronc C310A was synchronously

242 expressed and purified in E. coli (Fig. 3). However, the SIDronc C310A did not autoprocess

243 between the large subunit and small subunit, and we detected one major band that was supposed

244 to be the full length of SIDronc in purified SIDronc C310A boring a C-terminal His-tag. These

245 results indicate that SIDronc's caspase activity was essential for autocatalytic cleavage and that

246 SlDronc possessed caspase activity.

247 Recombinant SIDronc's strong activity on initiator caspase substrates

248 Synthetic caspase substrates have been widely used to identify caspase activity (Pop et al. 2008;

249 Talanian et al. 1997). To verify that SIDronc indeed has enzymatic activity, we measured the

250 catalytic activities of recombinant SIDronc with a C-terminal His-tag on 13 kinds of caspase

251 substrates. Comparing to the control, S1Dronc with a C-terminal His-tag showed dramatic

252 activities on Ac-VEID-AFC, Ac-LETD-AFC, Ac-IETD-AFC, Ac-LEHD-AFC, Ac-AEVD-AFC, 
254 showed no activity on the selected substrates (Fig. 4B). Catalytic site mutation completely

inhibited SIDronc activity, suggesting that wild type S1Dronc activities on caspase substrates rely

256

on caspase activity. This is consistent with the fact that SlDronc shows a high sequence

257

similarity to initiator caspase, and SIDronc showed the strongest activity against Ac-VEID-AFC,

a preferential substrate of initiator caspase. These data suggest that SIDronc may be an initiator

caspase.

\section{SIDronc's in vitro cleavage and activation of Sl-caspase-1}

It is widely known that initiator caspase can cleave effector caspase to activate effector caspase.

To determine whether S1Dronc can cleave recombinant S1-caspase-1 (an effector caspase in $S$.

littoralis), we expressed and purified S1-caspase-1 and S1-caspase-1 C178A (a catalytic site

to detect cleavage by SIDronc. Recombinant S1Dronc was incubated with Sl-caspase-1 C178A at

$37^{\circ} \mathrm{C}$ for $3 \mathrm{~h}$, and we analyzed the incubation mixture using western blotting and an Anti-Sf-

caspase-1 antibody. The result showed that S1-caspase-1 C178A was cleaved by S1Dronc, but not

by SIDronc C310A (Fig. 5A), suggesting that SIDronc could cleave Sl-caspase-1 directly in vitro and that SIDronc's caspase activity was necessary. Additionally, we found that incubation with

S1Dronc significantly increased the enzymatic activity of Sl-caspase-1 on Ac-DEVD-AFC (Fig.

5B), further confirming that SIDronc can activate Sl-caspase-1. Therefore, we suggest that

S1Dronc may function as an initiator caspase in the $S$. littoralis apoptotic pathway.

\section{Overexpression of SIDronc-induced apoptosis in SL2 cells}


274 To determine whether S1Dronc functions as an apoptotic caspase, we transfected plasmids that

275 either expressed wild type S1Dronc or the catalytic site mutant S1Dronc C310A with a C-terminal

276 Flag-tag, into SL2 cells, and we observed the cells across multiple intervals. Untreated cells and

277 cells transfected with plasmid that expressed C-terminally Flag-tagged GFP were used as

278

279

280

281

282

283

284

285

286

287

288

289

290

291

292

293

294

controls. At 24 h post transfection, we observed apoptosis in SL2 cells that expressed S1Dronc,

only slight apoptosis in SL2 cells that expressed SIDronc C310A, and no apoptosis in mock-

treated cells or cells that expressed GFP (Fig. 6A). Moreover, lysate prepared from the cells that

transiently expressed SIDronc showed significantly increased caspase activity on Ac-DEVD-

AFC when compared to cells that transiently expressed GFP (Fig. 6B), which is consistent with

the morphological results described previously. Western blot analysis that used an antibody

against Flag-tag showed that the full-length SIDronc's protein level was significantly lower than

that of S1Dronc C310A, suggesting that wild type S1Dronc is cleaved when transiently expressed

in SL2 cells (Fig. 6C). Western blot analysis using an antibody against Sf-caspase-1 showed that

the full-length S1-caspase-1 protein level was lower in cells that overexpressed SIDronc

compared to cells that overexpressed GFP (Fig. 6C). Taken together, these findings indicate that

apoptosis in SL2 cells was induced by SIDronc overexpression, and that SIDronc caspase activity was required to induce apoptosis. Therefore, SIDronc may be an apoptotic initiator caspase in

SL2 cells.

\section{Sldronc knockdown decreased apoptosis induced by UV irradiation in SL2 cells}

In order to verify SIDronc's function during apoptosis, we used dsRNA to knock down Sldronc expression. First, we proved that the prepared dsRNA against Sldronc (Sldronc-dsRNA) could 
295

296

297

298

299

300

301

302

303

304

305

306

307

308

309

310

311

312

313

314

315

successfully knock down endogenous Sldronc expression $24 \mathrm{~h}$ and $48 \mathrm{~h}$ after transfection (Fig.

7A). Next, we transfected SL2 cells with Sldronc-dsRNA for 24 h, followed by UV irradiation.

The SL2 cells transfected with dsRNA against $g f p$ ( $g f p$-dsRNA) and the cells transfected with

dsRNA against Sl-caspase-1 (Sl-caspase-1-dsRNA) were used as controls. We observed

decreased apoptosis in cells transfected with Sldronc-dsRNA or Sl-caspase-1-dsRNA compared

to cells transfected with $g f p$-dsRNA (Fig. 7B). We harvested the SL2 cells and analyzed the cell

lysates using caspase activity assay and western blotting. Consistent with the morphologic

results, the caspase activity assay showed decreased activity on Ac-DEVD-AFC in cells

transfected with Sldronc-dsRNA or Sl-caspase-1-dsRNA when compared to cells transfected

with $g f p$-dsRNA and treated with UV irradiation (Fig. 7C). The western blotting results showed

that Sldronc knockdown significantly decreased Sl-caspase-1 cleavage induced by UV

irradiation treatment in SL2 cells (Fig. 7D). These results confirmed S1Dronc's involvement in

apoptosis induced by UV irradiation in SL2 cells.

\section{Increase in processed SIDronc forms in the presence of $\mathrm{N}$-terminally truncated SIIAP}

IAP is vital in the regulation of apoptosis, and it is known to suppress caspase-mediated

apoptosis in D. melanogaster, B. mori, and S. frugiperda (Hamajima et al. 2016; Kaiser et al.

1998; Muro et al. 2002). To test the effects of SIIAP on SIDronc, we co-expressed SIIAP with

S1Dronc in SL2 cells. When S1Dronc containing C-terminal Flag-tag was co-expressed with

SIIAP, western blotting that utilized antibodies against Flag-tag only detected a decrease in full-

length SIDronc, but not in processed SIDronc. In order to distinguish between Pro+LS (39 kDa)

and LS+SS (36 kDa) detected by western blotting utilizing an anti-SIDronc antibody, we co- 
expressed N-terminally HA-tagged SIDronc with C-terminally Flag-tagged SIIAP in SL2 cells.

317 Western blotting using an antibody against SIDronc showed that the processed SlDronc protein

318 levels increased while the full-length S1Dronc protein levels decreased when co-expressed with

319 SIIAP. The processed form of $39 \mathrm{kDa}$ corresponded to the SlDronc form composed of the

prodomain and large subunit (Pro+LS), and the processed form of $24 \mathrm{kDa}$ corresponded to the

large subunit of SlDronc (LS) (Fig. 8A, first panel). Western blotting using an antibody against

HA-tag also showed that the full-length SIDronc protein level decreased and the processed

SIDronc protein levels increased when co-expressed with SIIAP (Fig. 8A, second panel).

Western blotting using an antibody against SfIAP showed that SIIAP mainly existed as cleaved

SIIAP (about $40 \mathrm{kDa}$ ) when co-expressed with S1Dronc in SL2 cells (Fig. 8A, third panel).

To further determine which portion of SIIAP functioned on SIDronc, we replaced the predicted

cleavage site D87 with Ala (A) to avoid N-terminally truncation, and replaced destabilizing

degron N88 at the N-terminus of cleaved SIIAP with Gly $(\mathrm{G})$, a stabilizing residue in the N-end

rule degradation pathway. When expressed in SL2 cells, the D87A mutation blocked cleavage,

indicated by the absence of cleaved SIIAP (40 kDa). N88G mutation stabilized the cleaved

SIIAP (Fig. 8B), confirming that SIIAP was cleaved at the site D87 in the N-terminal and that the

cleaved SIIAP was degraded by the N-end rule degradation pathway. When co-expressed with

S1Dronc in SL2 cells, the D87A mutant failed to increase the processed SIDronc protein levels,

while the N88G mutant significantly increased the processed S1Dronc protein levels (Fig. 8C).

These results suggest that SIIAP was cleaved at aspartate residue D87 in the N-terminal, the 
337 forms increased in the presence of N-terminally truncated SIIAP.

338 SIDronc inhibition by $\mathbf{P 4 9}$

339 P49, a baculoviral apoptosis inhibitor in SpliNPV, inhibits caspase activity of initiator caspases.

340 P35, a baculoviral apoptosis inhibitor in AcMNPV, inhibits the caspase activity of effector

341 caspases (Jabbour et al. 2002; Zoog et al. 2002). To determine whether S1Dronc was regulated by

342 P49, we tested the caspase activities of recombinant S1Dronc that had been expressed and

343 purified in E. coli by incubating them with increasing amounts of P49. The results showed that

344 P49 inhibited S1Dronc caspase activity in a dose-dependent manner (Fig. 9A). Using a 1:1

345 P49:S1Dronc ratio, we reduced SIDronc's enzymatic activity by 54\%. S1Dronc activity was

346 reduced by 97\% when the ratio was 4:1. To further test P49's effect on SIDronc in SL2 cells, we

347 co-expressed S1Dronc with increasing amounts of P49. Western blot analysis showed that the

348 full-length S1Dronc protein level increased gradually with increasing amounts of P49 when co-

349 expressed with S1Dronc (Fig. 9B). Protein grayscale analysis of full-length S1-caspase-1 showed

350 that the protein levels also increased slightly (Fig. 9C). These data indicate that SIDronc was

351 inhibited by P49.

352 Discussion

Dronc has proved to be an essential initiator caspase as it cleaves and activates effector caspases

to cleave downstream proteins in the apoptotic pathway, ultimately leading to apoptosis (Daish et

al. 2004; Dorstyn et al. 1999; Quinn et al. 2000). Dronc homologs from several Lepidopteran

insects have been studied, including BmDronc, LdDronc, and SfDronc (Huang et al. 2013;

Kitaguchi et al. 2013; Suganuma et al. 2011). In this study, we identified SIDronc, the first $S$. 
littoralis initiator caspase. SIDronc possesses typical initiator caspase features and was shown to induce apoptosis in SL2 cells.

Both mammalian and Drosophila caspases are subject to IAP regulation, and caspase ubiquitylation by IAPs can be either degradative or nondegradative (Darding \& Meier 2012;

Muro et al. 2002; Vaux \& Silke 2005). In degradative ubiquitylation, caspases are degraded by

proteasome. While nondegradative ubiquitylation does not lead to the degradation of caspases, it has been proposed that ubiquitin ( $\mathrm{Ub}$ ) can sterically occlude substrate entry and cause a conformational change in caspases by reducing their catalytic processivity (Ditzel et al. 2008).

Several studies have shown that ubiquitylated, full-length Dronc mediated by DIAP1 can be initially degraded by proteasome (Chai et al. 2003; Muro et al. 2002), while other researchers have found that processed and activated Dronc in the Dark apoptosome is degraded in a DIAP1dependent manner (Lee et al. 2011; Shapiro et al. 2008). Notably, both cBm-IAP1 depletion and overexpression can stabilize the cleaved form of Bm-Dronc, despite the opposite effect occurring during apoptosis in BM-N cells (Hamajima et al. 2016). In this study, SIIAP overexpression also increased the cleaved SIDronc protein levels (Pro+LS and LS) and decreased the full-length

SIDronc protein levels (Fig. 8A). This decrease observed in full-length SIDronc may be due to elevated ubiquitylation by the overexpressed SIIAP, but the mechanism by which the cleaved

SIDronc protein levels increased (Pro+LS and LS) requires further study. pathway. After receiving the apoptotic signal, SIDronc functions as an initiator caspase that 
379

380

substrates that serves as the executioner of apoptosis in SL2 cells. SIIAP functions as the last line of defense against apoptosis. The identification of SIDronc will facilitate further studies on $S$.

littoralis' apoptosis mechanism and baculovirus infection-induced apoptosis.

\section{Conclusion}

We identified that SIDronc, an initiator caspase in S. littoralis, can cleave and activate effector caspases. SIDronc overexpression induced apoptosis in SL2 cells, and Sldronc knockdown decreased apoptosis induced by UV irradiation. Our results indicate that SIDronc is an apoptotic initiator caspase in S. littoralis. Additionally, we found that processed forms of SIDronc increased in the presence of N-terminally truncated SIIAP and that SIDronc was inhibited by P49. This study will contribute to the elaboration of $S$. littoralis' apoptotic pathway and may facilitate future studies on baculovirus infection-induced apoptosis.

\section{Acknowledgements}

We are grateful to Dr. Nor Chejanovsky (Agricultural Research Organization, Volcani Center, Israel) for providing SL2 cells and antibodies against Sf-caspase-1.

\section{References}

Chai J, Yan N, Huh JR, Wu JW, Li W, Hay BA, and Shi Y. 2003. Molecular mechanism of Reaper-Grim-Hid-mediated suppression of DIAP1-dependent Dronc ubiquitination. Nat Struct Biol 10:892-898.

Chejanovsky N, and Gershburg E. 1995. The Wild-Type Autographa-Californica Nuclear Polyhedrosis-Virus Induces Apoptosis Of Spodoptera-Littoralis Cells. Virology 209:519-525.

Clem RJ. 2001. Baculoviruses and apoptosis: the good, the bad, and the ugly. Cell Death Differ 8:137-143.

Cohen GM. 1997. Caspases: the executioners of apoptosis. Biochem J 326 ( Pt 1):1-16.

Cooper DM, Granville DJ, and Lowenberger C. 2009. The insect caspases. Apoptosis 14:247-256.

Courtiade J, Pauchet Y, Vogel H, and Heckel DG. 2011. A comprehensive characterization of the caspase gene family in insects from the order Lepidoptera. BMC Genomics 12:357. 
Daish TJ, Mills K, and Kumar S. 2004. Drosophila caspase DRONC is required for specific developmental cell death pathways and stress-induced apoptosis. Dev Cell 7:909-915.

Darding M, and Meier P. 2012. IAPs: guardians of RIPK1. Cell Death Differ 19:58-66.

Degterev A, Boyce M, and Yuan J. 2003. A decade of caspases. Oncogene 22:8543-8567.

Deveraux QL, Takahashi R, Salvesen GS, and Reed JC. 1997. X-linked IAP is a direct inhibitor of cell-death proteases. Nature 388:300-304.

Ditzel M, Broemer M, Tenev T, Bolduc C, Lee TV, Rigbolt KT, Elliott R, Zvelebil M, Blagoev B, Bergmann A, and Meier P. 2008. Inactivation of effector caspases through nondegradative polyubiquitylation. Mol Cell 32:540-553.

Ditzel M, Wilson R, Tenev T, Zachariou A, Paul A, Deas E, and Meier P. 2003. Degradation of DIAP1 by the N-end rule pathway is essential for regulating apoptosis. Nat Cell Biol 5:467-473.

Donepudi M, Mac Sweeney A, Briand C, and Grutter MG. 2003. Insights into the regulatory mechanism for caspase-8 activation. Mol Cell 11:543-549.

Dorstyn L, Colussi PA, Quinn LM, Richardson H, and Kumar S. 1999. DRONC, an ecdysone-inducible Drosophila caspase. Proc Natl Acad Sci U S A 96:4307-4312.

Dorstyn L, and Kumar S. 2008. A biochemical analysis of the activation of the Drosophila caspase DRONC. Cell Death Differ 15:461-470.

Duprez L, Wirawan E, Vanden Berghe T, and Vandenabeele P. 2009. Major cell death pathways at a glance. Microbes Infect 11:1050-1062.

Elmore S. 2007. Apoptosis: a review of programmed cell death. Toxicol Pathol 35:495-516.

Galluzzi L, Lopez-Soto A, Kumar S, and Kroemer G. 2016. Caspases Connect Cell-Death Signaling to Organismal Homeostasis. Immunity 44:221-231.

Gibbs DJ, Bacardit J, Bachmair A, and Holdsworth MJ. 2014. The eukaryotic N-end rule pathway: conserved mechanisms and diverse functions. Trends Cell Biol 24:603-611.

Hamajima R, Iwamoto A, Tomizaki M, Suganuma I, Kitaguchi K, Kobayashi M, Yamada H, and Ikeda M. 2016. Functional analysis of inhibitor of apoptosis 1 of the silkworm Bombyx mori. Insect Biochem Mol Biol.

Hay BA, and Guo M. 2006. Caspase-dependent cell death in Drosophila. Annu Rev Cell Dev Biol 22:623-650.

Hill DS. 1987. Agricultural insect pests of temperate regions and their control. Cambridge Cambridgeshire ; New York: Cambridge University Press.

Huang N, Civciristov S, Hawkins CJ, and Clem RJ. 2013. SfDronc, an initiator caspase involved in apoptosis in the fall armyworm Spodoptera frugiperda. Insect Biochem Mol Biol 43:444-454.

Jabbour AM, Ekert PG, Coulson EJ, Knight MJ, Ashley DM, and Hawkins CJ. 2002. The p35 relative, p49, inhibits mammalian and Drosophila caspases including DRONC and protects against apoptosis. Cell Death Differ 9:1311-1320.

Kaiser WJ, Vucic D, and Miller LK. 1998. The Drosophila inhibitor of apoptosis D-IAP1 suppresses cell death induced by the caspase drICE. FEBS Lett 440:243-248.

Kitaguchi K, Hamajima R, Yamada H, Kobayashi M, and Ikeda M. 2013. Cloning and functional characterization of the Lymantria dispar initiator caspase dronc. Biochem Biophys Res Commun 436:331-337.

Kumar S, and Doumanis J. 2000. The fly caspases. Cell Death Differ 7:1039-1044.

Lee MJ, and Anstee JH. 1995. Endoproteases From the Midgut Of Larval Spodoptera-Littoralis Include a Chymotrypsin-Like Enzyme with an Extended Binding-Site. Insect Biochem Mol Biol 25:49-61.

Lee TV, Fan Y, Wang S, Srivastava M, Broemer M, Meier P, and Bergmann A. 2011. Drosophila IAP1-mediated 
ubiquitylation controls activation of the initiator caspase DRONC independent of protein degradation. PLOS Genet 7:e1002261.

Li J, and Yuan J. 2008. Caspases in apoptosis and beyond. Oncogene 27:6194-6206.

Liu Q, Qi Y, and Chejanovsky N. 2005. Spodoptera littoralis caspase-1, a Lepidopteran effector caspase inducible by apoptotic signaling. Apoptosis 10:787-795.

Lo YC, Lin SC, Yang CY, and Tung JY. 2015. Tandem DEDs and CARDs suggest novel mechanisms of signaling complex assembly. Apoptosis 20:124-135.

Mialhe E, Quiot JM, and Paradis S. 1984. Etablissement de deux lignees cellulaires deSpodoptera littoralis [Lep.: Noctuidae], permissives pour des virus susceptibles d'etre utilises en lutte microbiologique. Entomophaga 29:347-350.

Muro I, Hay BA, and Clem RJ. 2002. The Drosophila DIAP1 protein is required to prevent accumulation of a continuously generated, processed form of the apical caspase DRONC. J Biol Chem 277:49644-49650.

Muro I, Monser K, and Clem RJ. 2004. Mechanism of Dronc activation in Drosophila cells. J Cell Sci 117:5035-5041.

Pop C, and Salvesen GS. 2009. Human caspases: activation, specificity, and regulation. J Biol Chem 284:21777-21781.

Pop C, Salvesen GS, and Scott FL. 2008. Caspase assays: identifying caspase activity and substrates in vitro and in vivo. Methods Enzymol 446:351-367.

Pop C, Timmer J, Sperandio S, and Salvesen GS. 2006. The apoptosome activates caspase-9 by dimerization. Mol Cell 22:269-275.

Quinn LM, Dorstyn L, Mills K, Colussi PA, Chen P, Coombe M, Abrams J, Kumar S, and Richardson H. 2000. An essential role for the caspase dronc in developmentally programmed cell death in Drosophila. Journal Of Biological Chemistry 275:40416-40424.

Riedl SJ, and Shi Y. 2004. Molecular mechanisms of caspase regulation during apoptosis. Nat Rev Mol Cell Biol 5:897907.

Roy N, Deveraux QL, Takahashi R, Salvesen GS, and Reed JC. 1997. The c-IAP-1 and c-IAP-2 proteins are direct inhibitors of specific caspases. EMBO J 16:6914-6925.

Salvesen GS, and Abrams JM. 2004. Caspase activation - stepping on the gas or releasing the brakes? Lessons from humans and flies. Oncogene 23:2774-2784.

Salvesen GS, and Dixit VM. 1999. Caspase activation: the induced-proximity model. Proc Natl Acad Sci U S A 96:10964-10967.

Shapiro PJ, Hsu HH, Jung H, Robbins ES, and Ryoo HD. 2008. Regulation of the Drosophila apoptosome through feedback inhibition. Nat Cell Biol 10:1440-1446.

Suganuma I, Ushiyama T, Yamada H, Iwamoto A, Kobayashi M, and Ikeda M. 2011. Cloning and characterization of a dronc homologue in the silkworm, Bombyx mori. Insect Biochem Mol Biol 41:909-921.

Summers MD, and Smith GE. 1987. A Manual Of Methods for Baculovirus Vectors And Insect Cell-Culture Procedures. Texas Agricultural Experiment Station Bulletin:1-56.

Tait SWG, and Green DR. 2010. Mitochondria and cell death: outer membrane permeabilization and beyond. Nature Reviews Molecular Cell Biology 11:621-632.

Talanian RV, Quinlan C, Trautz S, Hackett MC, Mankovich JA, Banach D, Ghayur T, Brady KD, and Wong WW. 1997. Substrate specificities of caspase family proteases. J Biol Chem 272:9677-9682.

Tasaki T, Sriram SM, Park KS, and Kwon YT. 2012. The N-end rule pathway. Annu Rev Biochem 81:261-289.

Vaux DL, and Silke J. 2005. IAPs, RINGs and ubiquitylation. Nat Rev Mol Cell Biol 6:287-297. 
486

487

488

489

490

491

492

493

494

495

496

497

498

499
Vilaplana L, Pascual N, Perera N, and Belles X. 2007. Molecular characterization of an inhibitor of apoptosis in the Egyptian armyworm, Spodoptera littoralis, and midgut cell death during metamorphosis. Insect Biochem Mol Biol 37:1241-1248.

Wallach D, Kang TB, Dillon CP, and Green DR. 2016. Programmed necrosis in inflammation: Toward identification of the effector molecules. Science 352:aaf2154.

Watt W, Koeplinger KA, Mildner AM, Heinrikson RL, Tomasselli AG, and Watenpaugh KD. 1999. The atomicresolution structure of human caspase-8, a key activator of apoptosis. Structure 7:1135-1143.

Yan N, Wu JW, Chai J, Li W, and Shi Y. 2004. Molecular mechanisms of DrICE inhibition by DIAP1 and removal of inhibition by Reaper, Hid and Grim. Nat Struct Mol Biol 11:420-428.

Yang Z, Zhou K, Liu H, Wu A, Mei L, and Liu Q. 2016. SfDredd, a Novel Initiator Caspase Possessing Activity on Effector Caspase Substrates in Spodoptera frugiperda. PLoS One 11:e0151016.

Zoog SJ, Schiller JJ, Wetter JA, Chejanovsky N, and Friesen PD. 2002. Baculovirus apoptotic suppressor P49 is a substrate inhibitor of initiator caspases resistant to P35 in vivo. Embo Journal 21:5130-5140. 


\section{Figure 1}

SIDronc sequence analysis

SIDronc amino acid sequence is shown aligned to Dronc homologs including SfDronc, BmDronc, AeDronc and DmDronc. Uniform amino acid residues in five Droncs are shown in white on a black background, the amino acid residues identical in four Droncs are shown in black on a shaded background. The CARD domain, caspase P20 domain, caspase P10 domain, predicted $\alpha$-helices and $\beta$-sheets are marked above the sequence. $A$ box is used to outline the predicted catalytic site, and inverted triangles are used to line out cleavage sites at position E128 and D338. The alignment was performed using DNAMAN Version 7. InterProScan Version 5.0 and PsiPred Version 3.3 were used to predict the conserved motifs and secondary structure in SIDronc. 


\section{Figure 2}

SIDronc phylogenetic analysis

Amino acid sequence of SIDronc and 30 chosen insect caspases were utilized to biuld the phylogenetic tree using the neighbor-joining method by MEGA Version 5.05. The sequences contain the following: SIDronc, SI-caspase-1 and SI-caspase-3 in Spodoptera littoralis , Splicaspase-5 and Spli-caspase-6 in Spodoptera litura, Se-caspase-5 and Se-caspase-6 in Spodoptera exigua, Ha-caspase-5 and Ha-caspase-6 in Helicoverpa armigera, SfDredd, SfDronc and Sf-caspase-1 in Spodoptera frugiperda, Hv-caspase-6 in Heliothis virescens, Mscaspase- 6 in Manduca sexta, Gm-caspase- 6 in Galleria mellonella, Bm-caspase-1, BmICE, Bm-caspase-4, BmDronc and BmDredd in Bombyx mori, Ld-caspase-5 in Lymantria dispar , Pr-caspase-5 in Pieris rapae, AeDronc and AeDredd in Aedes aegypti, Dcp1, DECAY, DAMM, DrICE, STRICA, DmDronc and DmDredd in Drosophila melanogaster. Genbank accession numbers of above sequences are listed in Table 4. 


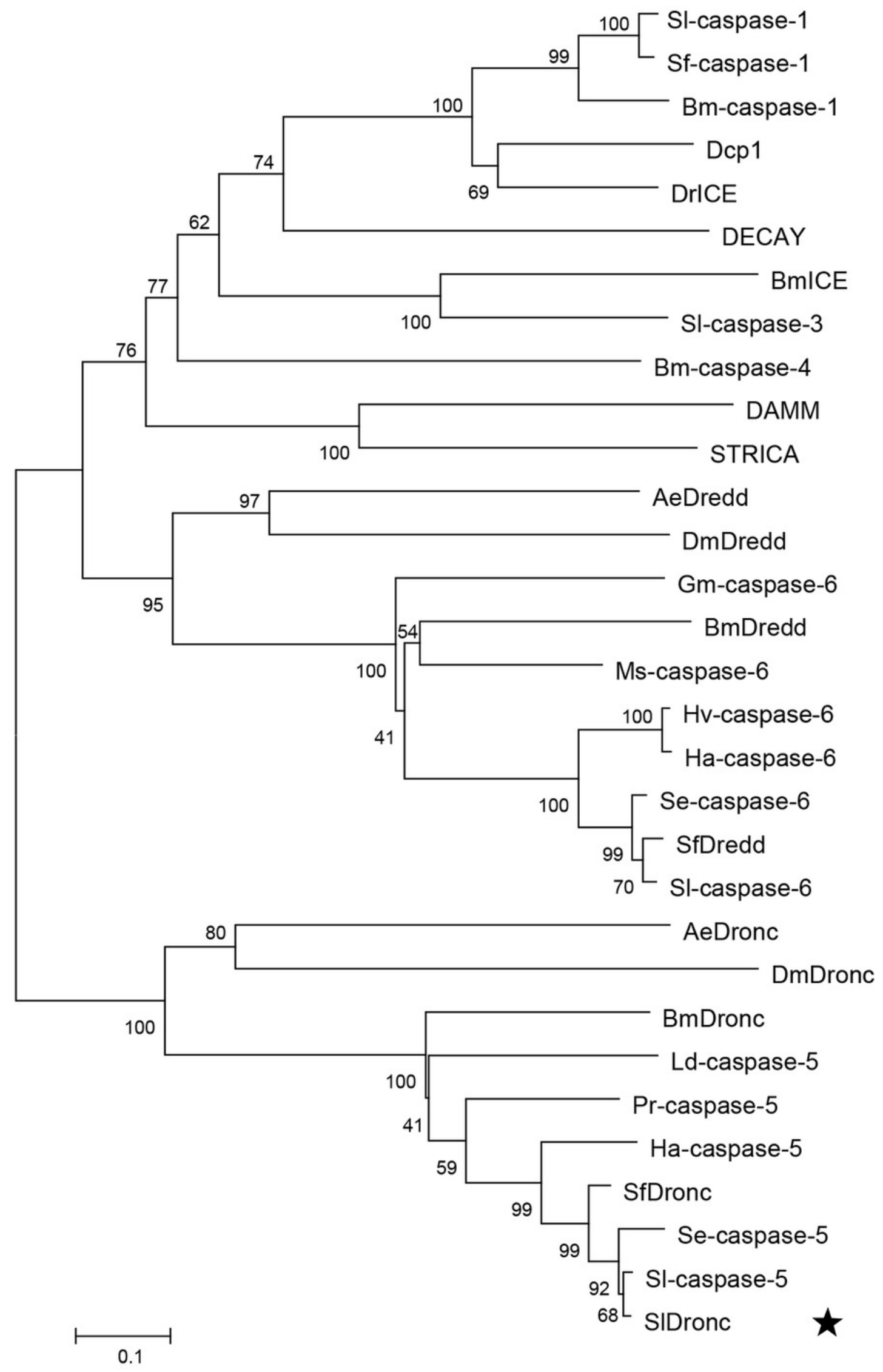




\section{Figure 3}

SIDronc autocatalytic cleavage in E. coli

Wild type SIDronc and the catalytic site mutant SIDronc C310A C-terminal His-tag were expressed in E. coli and purified proteins were detected by western blotting utilizing antibody against His-tag or SIDronc following SDS-PAGE. His represents His-tag , Pro represents prodomain, LS represents large subunit, SS represents small subunit. NSC represents Band might be due to non-specific cleavage by a bacterial protease. 


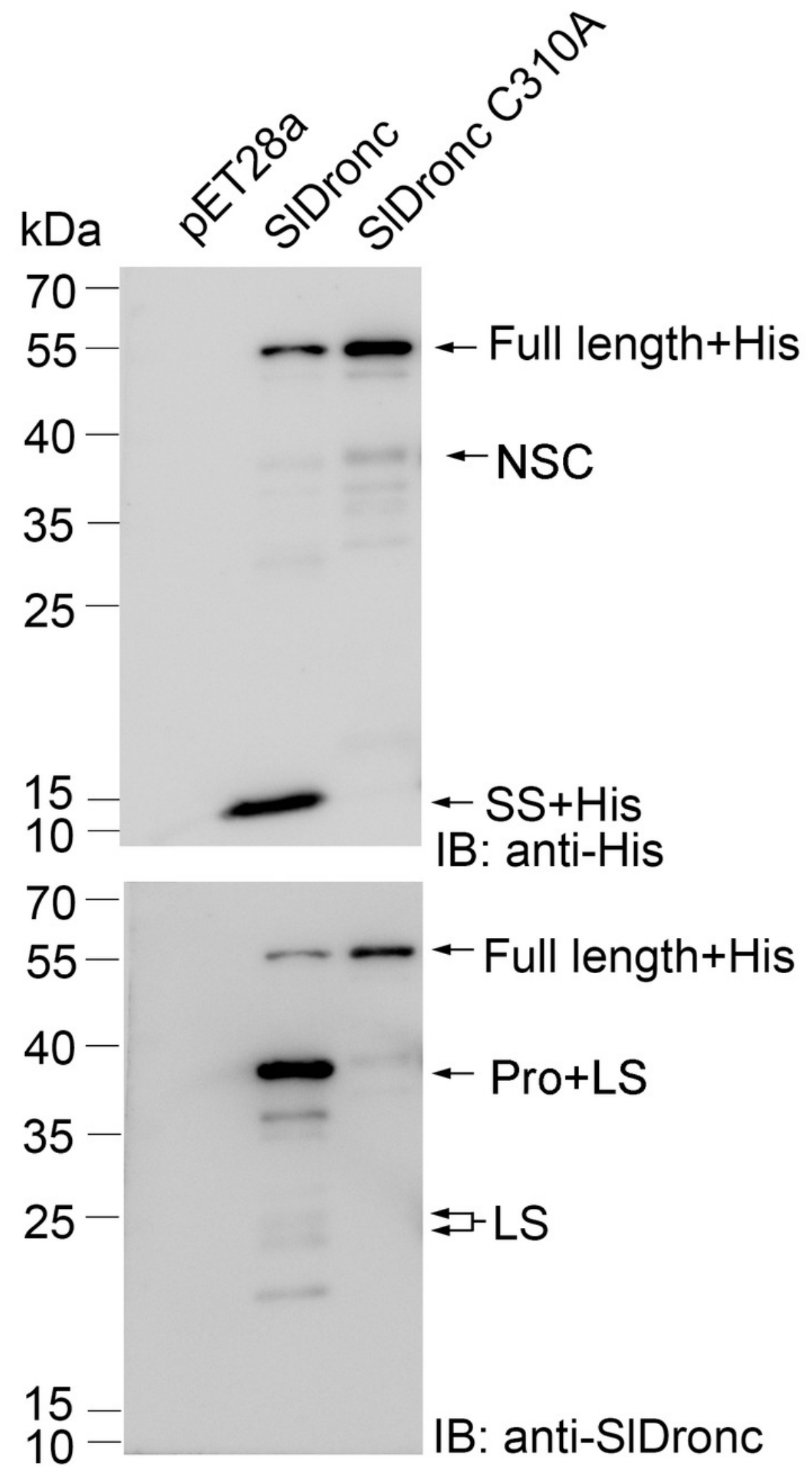




\section{Figure 4}

Recombinant SIDronc's strong activity on initiator caspase substrates

(A) and (B) Wild type SIDronc (A) and catalytic site mutant SIDronc C310A (B) containing a His-tag in C-terminal were incubated with 13 kinds of synthetic caspase substrates or 7 selected kinds of synthetic caspase substrates $(20 \mu \mathrm{M})$ respectively, and then the mixture were subjected to caspase activity assay. Changes in RFU (relative fluorescence units) per minute was used to show caspase activity. SD from three independent experiments were presented. 


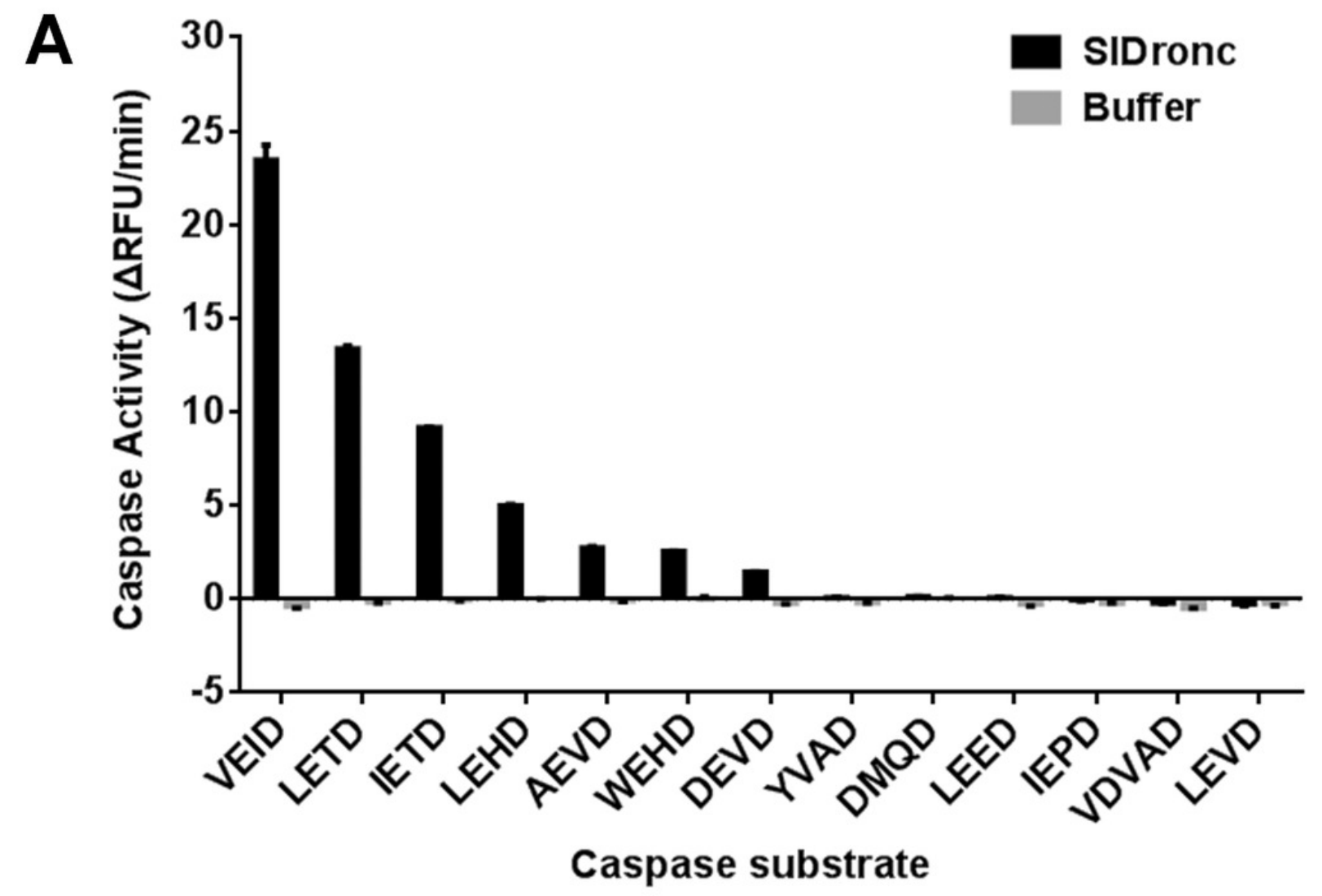

B

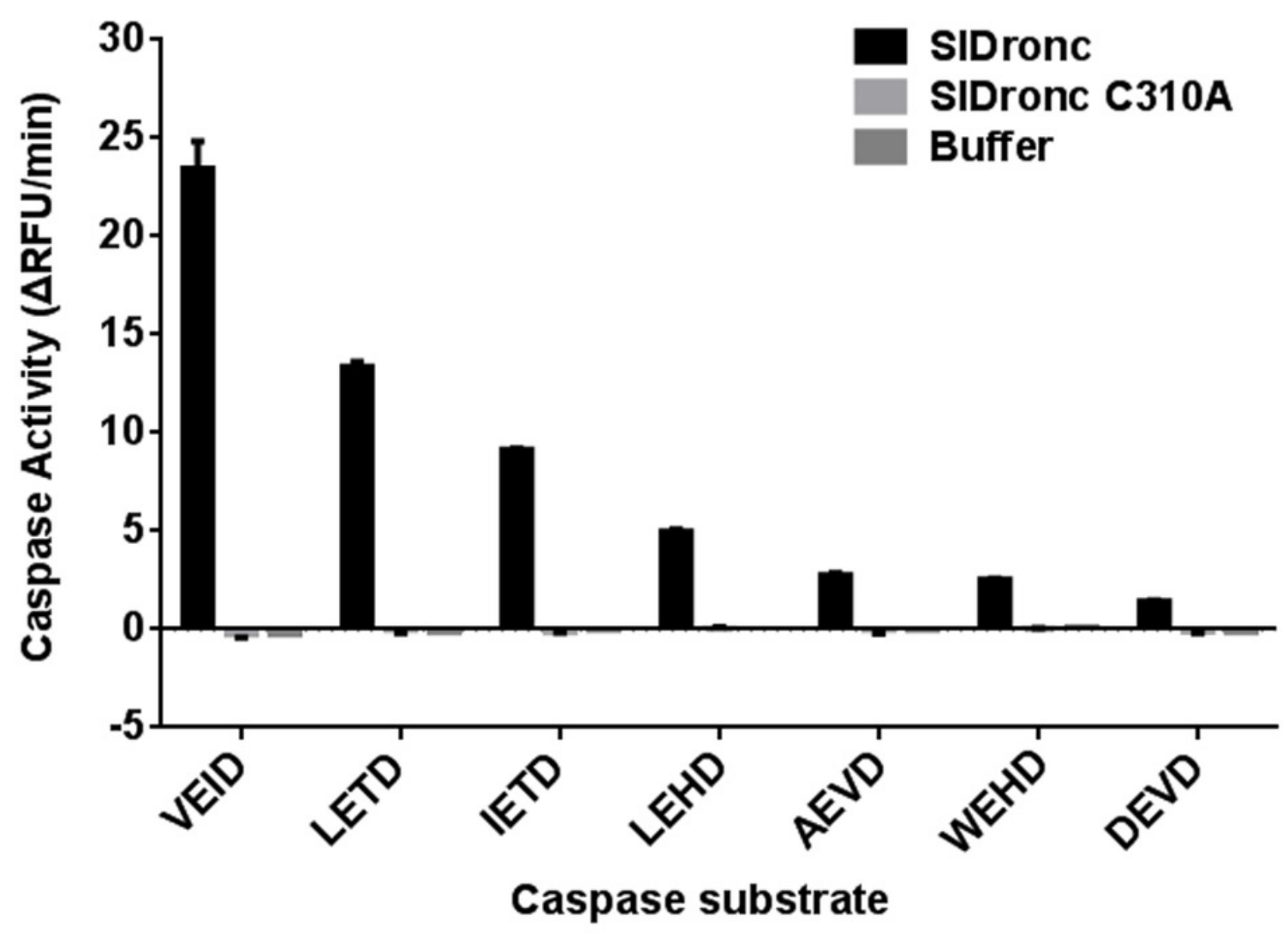




\section{Figure 5}

SIDronc's in vitro cleavage and activation of SI-caspase-1

(A) SI-caspase-1 C178A (600 nM) was incubated respectively with $600 \mathrm{nM}$ of SIDronc or SIDronc C310A with C-terminal His-tag at 37 ㅇ $\mathrm{C}$ for $3 \mathrm{~h}$ in Na-Citrate buffer, and then the mixtures were detected by western blotting using Anti-Sf-caspase-1 antibody . (B) SIcaspase-1 (5 nM) was incubated with buffer and SIDronc (240 nM) respectively. SI-caspase-1 C178A (5 nM) was incubated with SIDronc (240 nM). Then caspase activity of the mixtures were measured using synthetic caspase substrate Ac-DEVD-AFC (20 $\mu \mathrm{M})$. Caspase activity was indicated relative to that of SI-caspase-1 (5 nM) or Sf-caspase-1 (5 nM) incubated with buffer. SD from three independent experiments were presented, and statistical significance was analysied by $t$ test. 


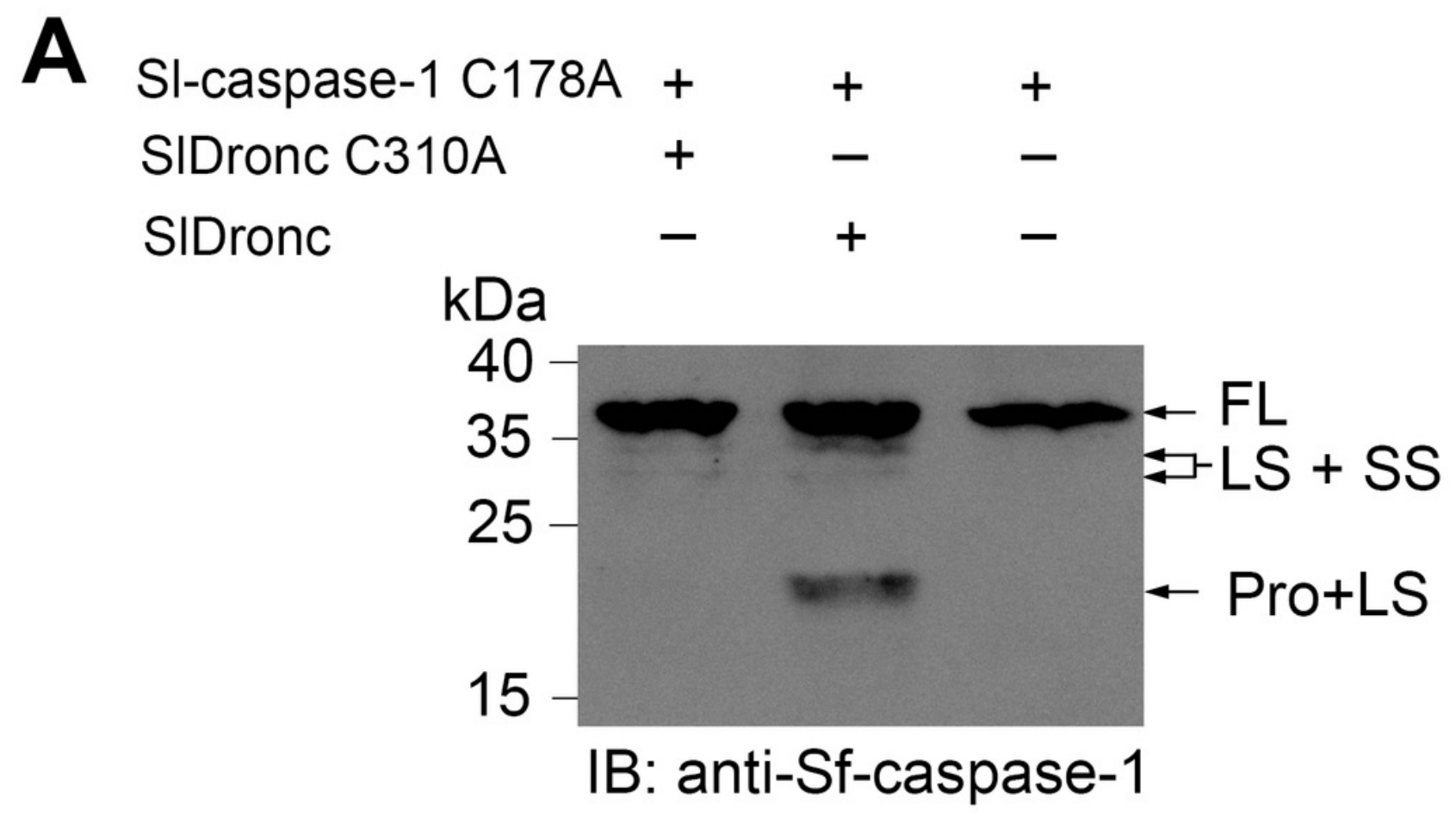

B

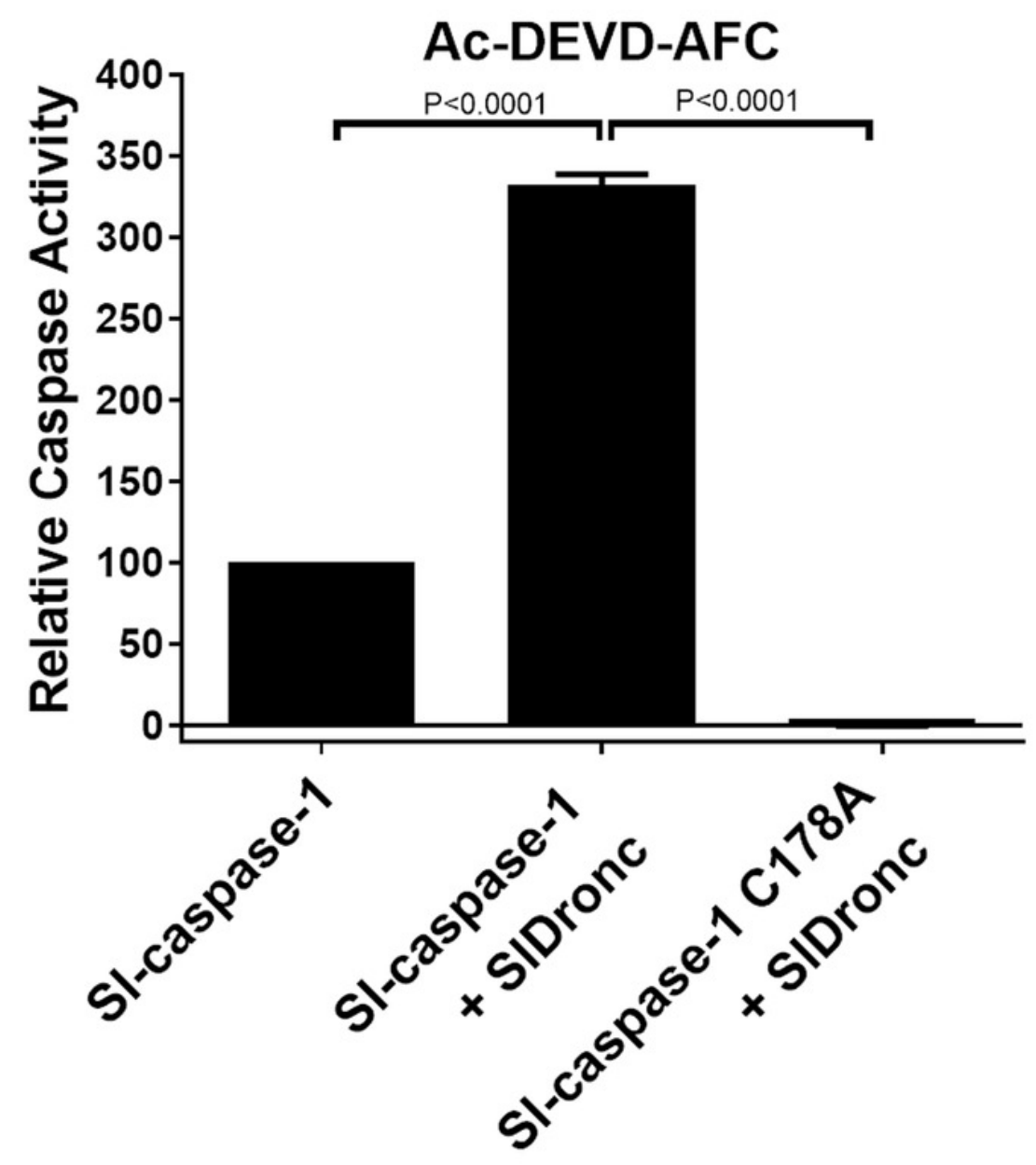




\section{Figure 6}

Overexpression of SIDronc-induced apoptosis in SL2 cells

SL2 cells were transfected separately with plasmids which express C-terminally Flag-tagged wild type SIDronc, catalytic site mutant SIDronc C310A and GFP for $24 \mathrm{~h}$ and subject to the following analysis. (A) SL2 cell pictures were taken (magnification $\times 200$ ), the scale bar represents $50 \mu \mathrm{m}$. Arrows were used to mark the apoptotic cells. (B) and (C) SL2 cells were harvested and the prepared cell lysates were subjected to caspase activity assay (B) or western blotting utilizing antibodies against Flag-tag, Sf-caspase-1 or $\beta$-actin (C). «: could be product of initiating from inner ATG. Changes in RFU (relative fluorescence units) per minute was used to show caspase activity. SD from three independent experiments were presented, and statistical significance was analysied by $t$ test. 
A

SL2 cells
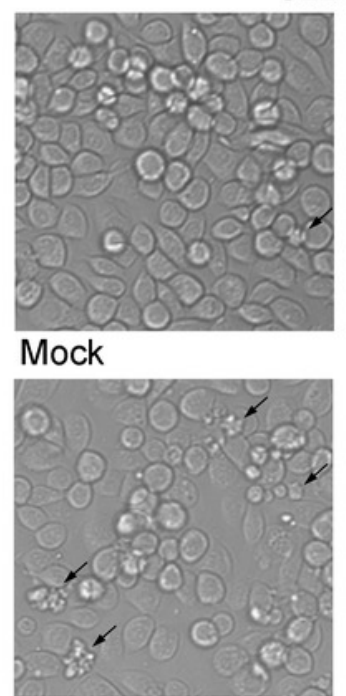

SIDronc

B

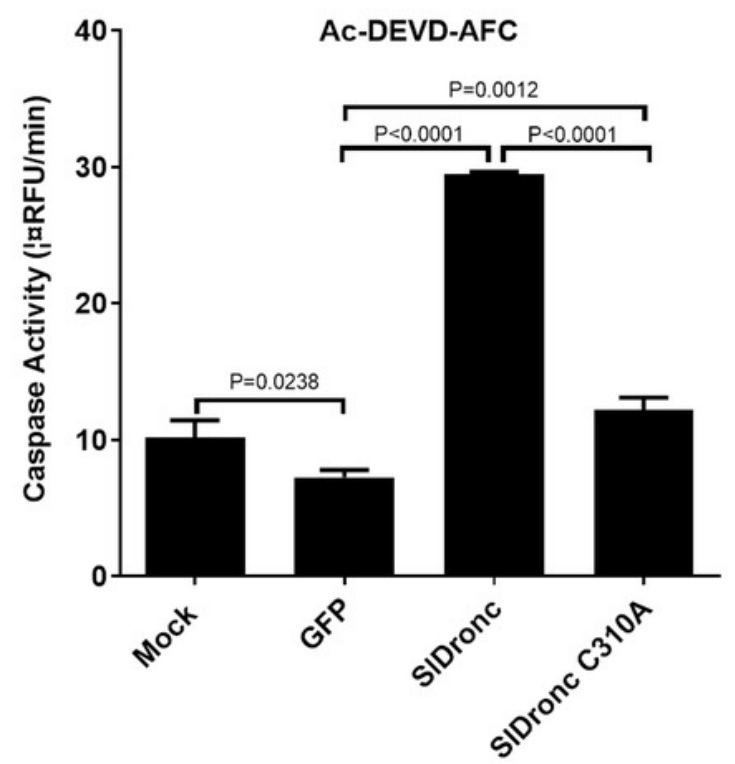

C

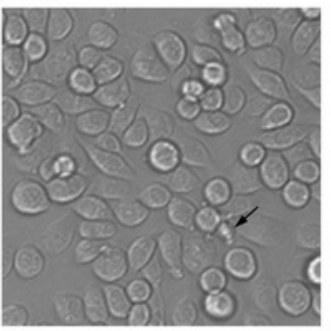

GFP

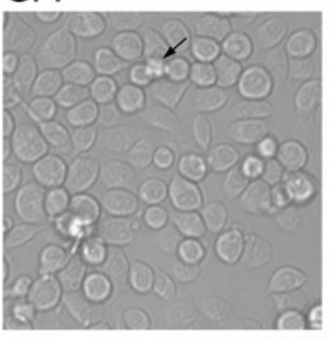

SIDronc C310A

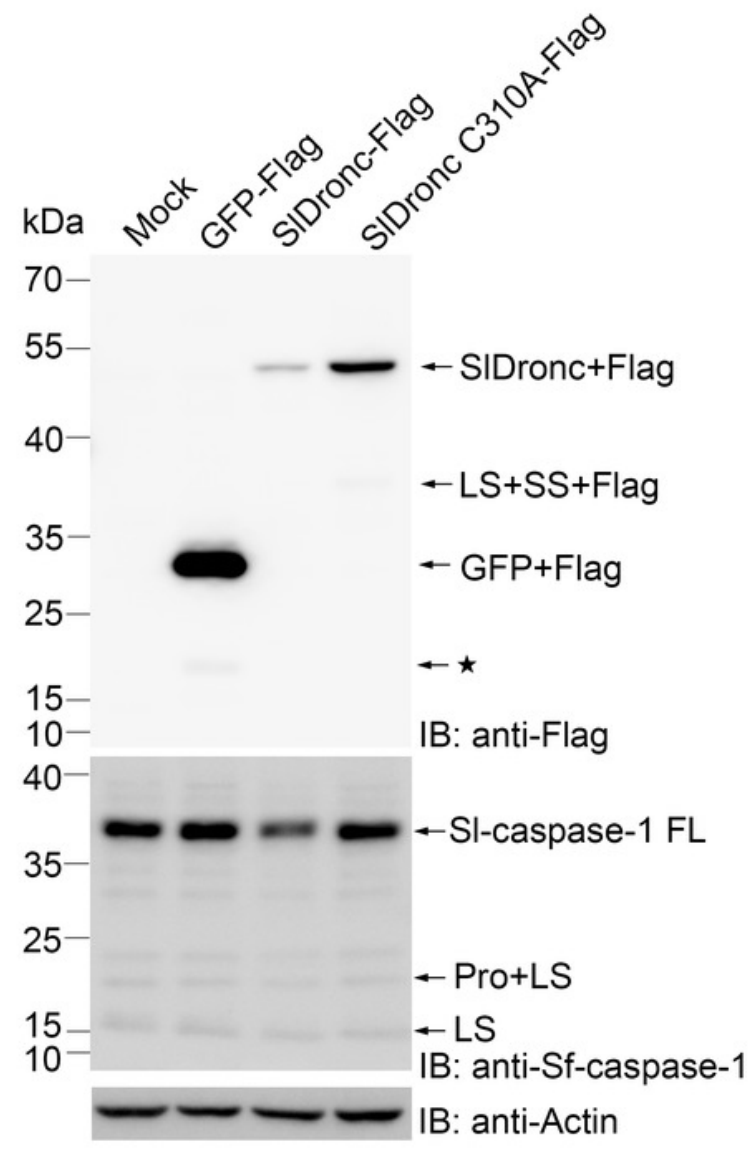




\section{Figure 7}

Sldronc knockdown decreased apoptosis induced by UV irradiation in SL2 cells

(A) SL2 cells were transfected with Sldronc-dsRNA or gfp-dsRNA. At $24 \mathrm{~h}$ and $48 \mathrm{~h}$ post transfection, cells were harvested and cell lysates were subjected to western blotting with antibody against SIDronc or $\beta$-actin. (B-D) SL2 cells were transfected with SIdronc-dsRNA, gfp-dsRNA or SI-caspase-1-dsRNA for $24 \mathrm{~h}$ and then treated with UV irradiation. At $24 \mathrm{~h}$ post UV treatment, SL2 cells were subjected to following analysis. (B) SL2 cell pictures were taken (magnification $\times 200$ ), and the scale bar represents $50 \mu \mathrm{m}$. (C) SL2 cells were harvested and caspase activity of the cell lysates were measured using Ac-DEVD-AFC. (D) The cell lysates were subjected to western blotting utilizing antibodies against SIDronc, Sf-caspase-1 or $\beta$ actin. Caspase activity was indicated relative to that of SL2 cells transfected with gfp-dsRNA and treated by UV. SD from three independent experiments were presented, and statistical significance was analysied by $t$ test. 
A

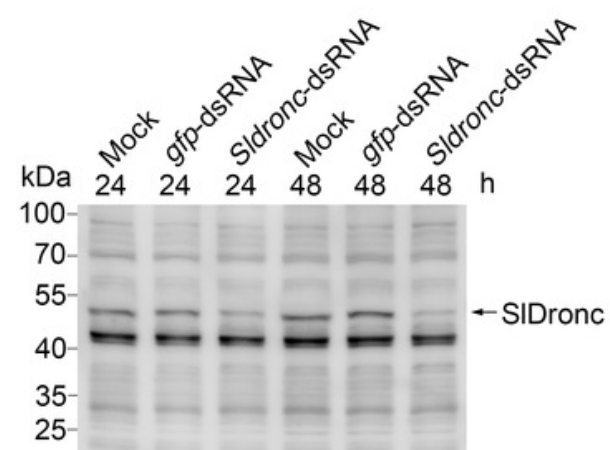

15

$----1 \mathrm{IB}$ : anti-Actin

C

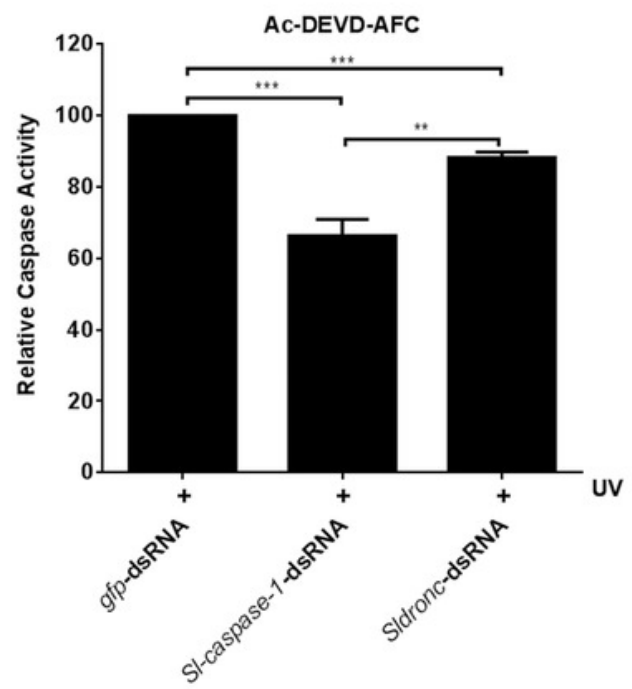

B
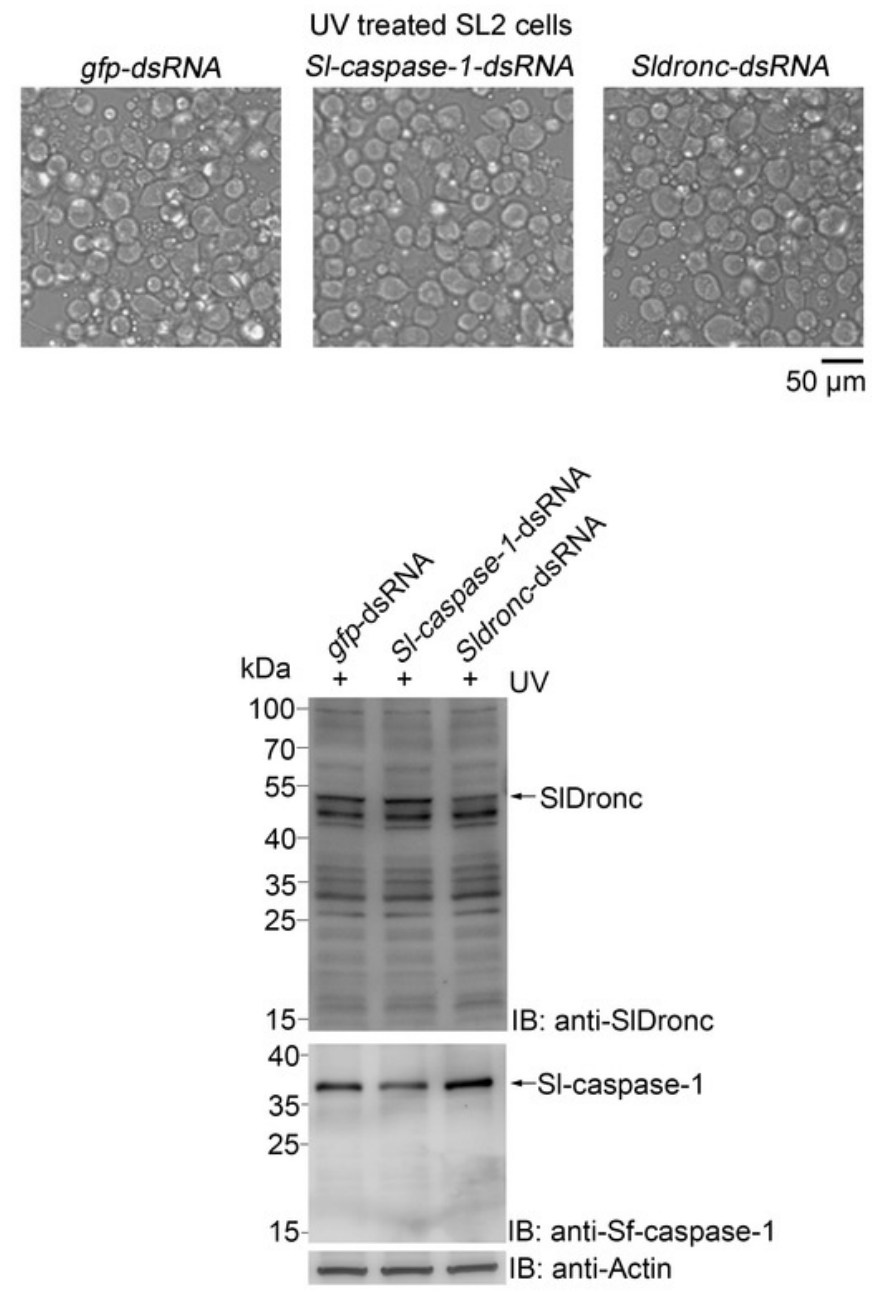


\section{Figure 8}

Increase in processed SIDronc forms in the presence of N-terminally truncated SIIAP

SL2 cells were transfected with plasmids respectively. Vector plasmid was used to make sure

$\mathrm{SL2}$ cells in each well were transfected with same amount of plasmid. At $24 \mathrm{~h}$ post

transfection, cells were harvested and cell lysates were subjected to western blotting. (A)

SL2 cells were transfected with plasmid expressing N-terminally HA-tagged SIDronc and plasmid expressing C-terminally Flag-tagged SIIAP, cells transfected with plasmid expressing SIDronc alone and cells mock treated were used as controls. (B) SL2 cells were transfected with plasmids expressing SIIAP WT, SIIAP D87A or SIIAP N88G respectively. (C) SL2 cells were cotransfected with plasmid expressing SIDronc and plasmid expressing SIIAP WT, SIIAP D87A or SIIAP N88G respectively, cells transfected with plasmid expressing SIDronc alone and cells mock treated were used as controls. A short vertical line indicates where lanes were removed and divided parts of the same Western blot image were joined together. 

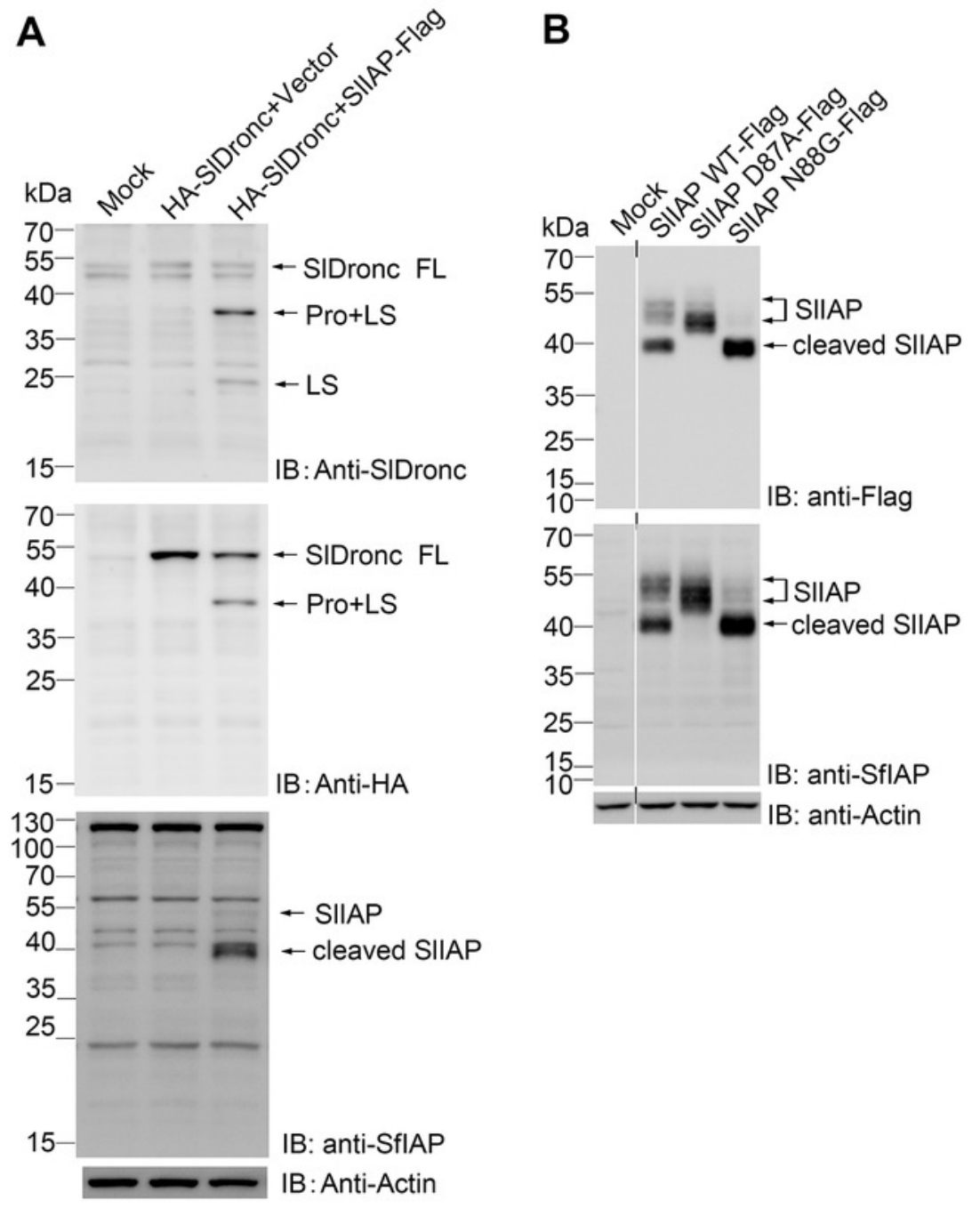

C

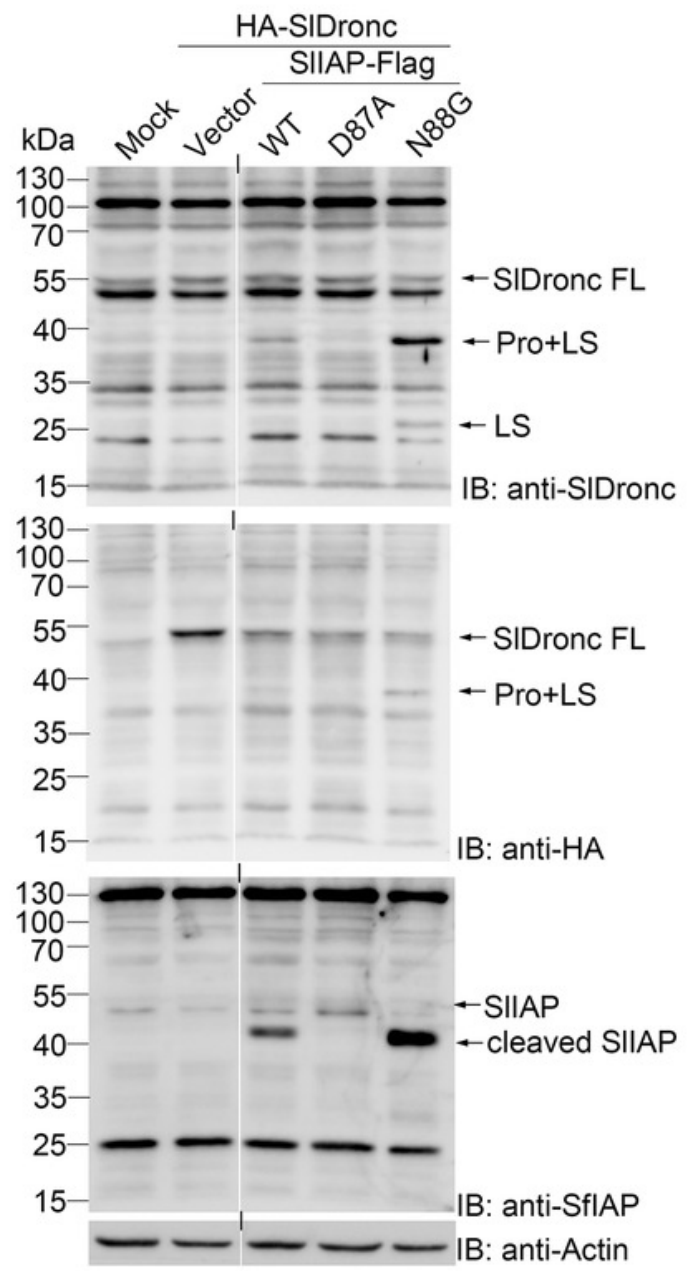




\section{Figure 9}

SIDronc inhibition by P49

(A) SIDronc (360 nM) was incubated with increasing amount of P49, and then the ability of the caspases to cleave Ac-VEID-AFC was determined. Caspase activity was indicated relative to that of SIDronc incubated with buffer. SD from three independent experiments were presented, and statistical significance was analysied by $t$ test . (B) SIDronc was co-expressed with increasing amount of P49. Plasmid expressing GFP was used to make sure SL2 cells in each well were transfected with same amount of plasmid. At $24 \mathrm{~h}$ post transfection, SL2 cells were harvested and cell lysates were analysied by western blotting utilizing antibodies against Flag-tag, Sf-caspase-1, His-tag or $\beta$-actin. (C) Protein grayscale analysis of full length SI-caspase-1 was performed using Quantity One. Band density was indicated relative to that of cells expressing SIDronc and GFP. 
A

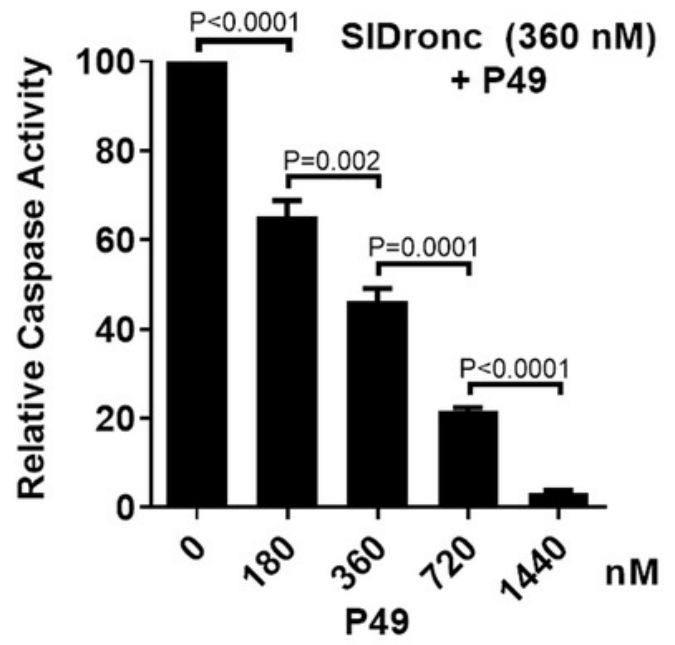

C

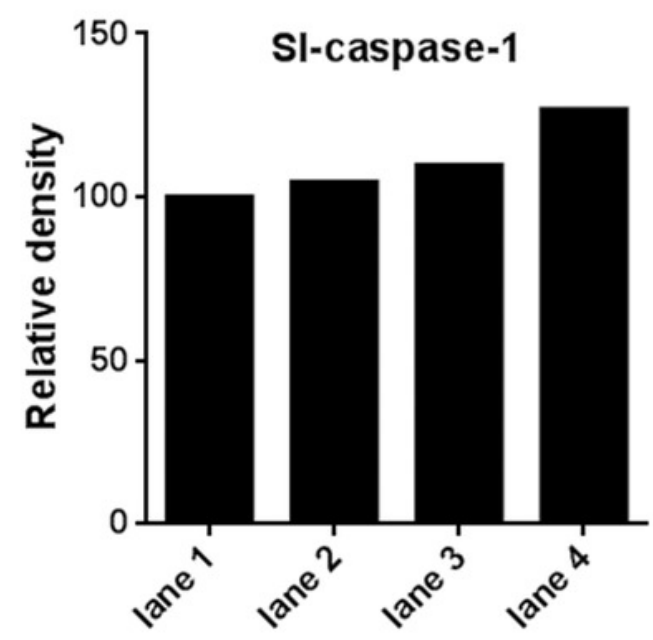

B

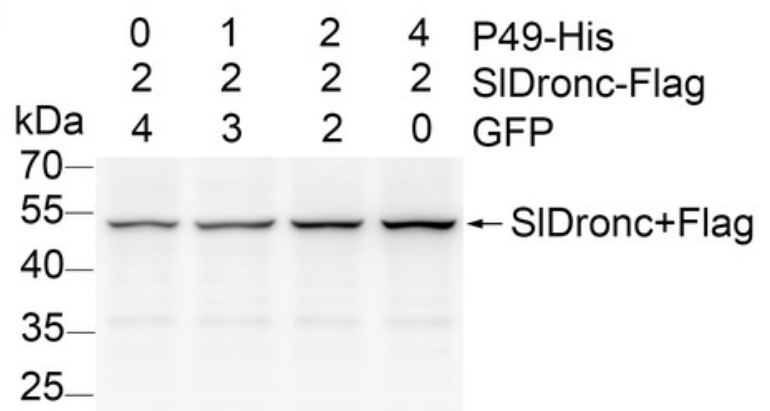

15 $10-$

40 35 25 15 70 55 40IB: anti-Flag 35 25IB: anti-His IB: anti-Actin 
Table $\mathbf{1}$ (on next page)

Primers used for RACE of SIDronc 
1 Table 1. Primers used for RACE of SIDronc.

\begin{tabular}{ll}
\hline Primer name & Primer sequence \\
\hline $1-17-5$ 'GSP1 & CCTCTTATTGAAGCCCCAGCGGTCCAC \\
$1-17-5$ 'NGSP1 & GTATGTCGGAGTAGAGCGGCGTCTGTC \\
$1-17-3$ 'GSP1 & GATTCCACCCCATTCCCCAACCTAAGC \\
$1-17-3$ 'NGSP1 & GATGAACTCGGCTTTCGTAGGCTCGG \\
\hline
\end{tabular}

2 
Table 2 (on next page)

Primers used for mutagenesis 
1 Table 2. Primers used for mutagenesis.

\begin{tabular}{ll}
\hline Primer name & Primer sequence \\
\hline SIDronc C310A(S) & CAAATGGCCAGAGGCAGCAGTG \\
SiDronc C310A(A) & CTGCTGCCTCTGGCCATTTGG \\
Sl-caspase-1 C178A(S) & GCTGCCCAAGGTGATAAATTG \\
Sl-caspase-1 C178A(A) & CTTGGGCAGCTTGAATAAAG \\
SIIAP D87A-AS & GTCGTGATTGGCGGTTTTATCGATG \\
SIIAP D87A-S & GATAAAACCGCCAATCACGACACC \\
SIIAP N88G-AS & GTCGTGACCGTCGGTTTTATCGATG \\
SIIAP N88G-S & GATAAAACCGACGGTCACGACACC \\
\hline
\end{tabular}

2 
Table 3 (on next page)

Primers used for dsRNA 
1 Table 3. Primers used for dsRNA.

\begin{tabular}{ll}
\hline Primer name & Primer sequence \\
\hline T7-SIDronc-dsRNA-F & TAATACGACTCACTATAGGGCATCCAAGCATTGTGCGAGGT \\
T7-SIDronc-dsRNA-R & TAATACGACTCACTATAGGGTGACTATTACCTGCTACTCTGTTA \\
T7-Sl-caspase-1-dsRNA-F & TAATACGACTCACTATAGGGCCATTATTTTCAATCACGAGCATTT \\
T7-Sl-caspase-1-dsRNA-R & TAATACGACTCACTATAGGGCTAGAGCTACTTTCTGACACACA \\
T7-GFP-dsRNA-F & TAATACGACTCACTATAGGGATGGTGAGCAAGGGCGAGGA \\
T7-GFP-dsRNA-R & TAATACGACTCACTATAGGGTTGAAGTTCACCTTGATGCC \\
\hline
\end{tabular}

2 
Table 4 (on next page)

Sequences used for alignments and phylogenetic tree 
1 Table 4. Sequences used for alignments and phylogenetic tree.

\begin{tabular}{|c|c|c|c|}
\hline Species & Name & Length & Genbank accession \\
\hline Aedes aegypti & AeDredd & 493 aа & ABI74776.1 \\
\hline Aedes aegypti & AeDronc & 449 aa & XP_001655433.1 \\
\hline Bombyx mori & Bm-caspase-1 & 293 aa & NP_001037050.1 \\
\hline Bombyx mori & Bm-caspase-3/a (BmICE) & 284 aa & ABC94941.1 \\
\hline Bombyx mori & Bm-caspase-4 & 497 aa & AEK71902.1 \\
\hline Bombyx mori & Bm-caspase-5 (BmDronc) & 438 aа & NP_001182396.1 \\
\hline Bombyx mori & Bm-caspase-6 (BmDredd) & 543 aa & BAF98475.1 \\
\hline Drosophila melanogaster & Dep1 & 323 aa & NP_476974.1 \\
\hline Drosophila melanogaster & DECAY & 308 aa & AAD54071.2 \\
\hline Drosophila melanogaster & DAMM & 255 aa & AAF58613.3 \\
\hline Drosophila melanogaster & DrICE & 339 aа & CAA72937.1 \\
\hline Drosophila melanogaster & STRICA (Dream) & 527 aа & AAF57292.2 \\
\hline Drosophila melanogaster & DmDredd & 494 aа & AAC33117.1 \\
\hline Drosophila melanogaster & DmDronc & 450 aа & AAD26625.1 \\
\hline Galleria mellonella & Gm-caspase-6 & 537 aa & AEK20837.1 \\
\hline Helicoverpa armigera & Ha-caspase-5 & 453 aа & AEK20835.1 \\
\hline Helicoverpa armigera & Ha-caspase-6 & 542 aa & AEK20838.1 \\
\hline Heliothis virescens & Hv-caspase-6 & 547 aa & HQ328982.1 \\
\hline Lymantria dispar & Ld-caspase-5 & 443 aа & BAL60586.1 \\
\hline Manduca sexta & Ms-caspase-6 & 543 aа & AEF30497.1 \\
\hline Pieris rapae & Pr-caspase-5 (partial) & 252 aa & AEK20836.1 \\
\hline Spodoptera exigua & Se-caspase-5 & 453 aa & AFX60235.1 \\
\hline Spodoptera exigua & Se-caspase-6 & 548 aa & AFO64608.1 \\
\hline Spodoptera frugiperda & Sf-caspase-1 & 299 aа & AAC47442.1 \\
\hline Spodoptera frugiperda & SfDronc & 447 aa & JX912275 \\
\hline Spodoptera frugiperda & SfDredd & 552 aa & AMR71144.1 \\
\hline Spodoptera littoralis & Sl-caspase-1 & 299 aa & AAO16241.1 \\
\hline Spodoptera littoralis & Sl-caspase-3 & 281 aa & AEK20824.1 \\
\hline Spodoptera litura & Spli-caspase-5 & 445 aa & AFJ04535.1 \\
\hline Spodoptera litura & Spli-caspase-6 & 522 aa & AFJ04536.1 \\
\hline
\end{tabular}

